\title{
Emboli characterization in Blood Mimicking Fluid circulation using parametric modeling
}

\author{
M. Biard, J-M. Girault, D. Kouamé, and F. Patat \\ LUSSI/GIP Ultrasons, \\ 2 bis boulevard Tonnellé \\ 37032 Tours, FRANCE \\ Email: biard_m@med.univ-tours.fr
}

\begin{abstract}
Cerebral microemboli remain a high part of all vascular cerebral accidents in western world. Thus detection, sizing and nature (particulate or gaseous) evaluation are three important tasks which are necessary to understand embolism and to perform correct diagnostics and even therapy. The problem of detection has been widely reported in many works. Our aim was here to address the emboli characterization, sizing and nature evaluation. This characterization is based on EBR (Embolus to Blood Ratio). EBR values were computed with two signal processing approaches: Fourier technique and Parametric modeling. Emboli were simulated with acrylic particles ranging from $220 \mu \mathrm{m}$ to $500 \mu \mathrm{m}$. With the smallest emboli, parametric approaches gave variance and standard deviation better than Fourier technique. In a second time, we use the same multifrequency approach to find frequencies triplet which assumed the best differentiation between gaseous and particulate emboli over a high range of diameters.
\end{abstract}

\section{INTRODUCTION}

Transcranial Doppler ultrasound (US) is the most commonly used technique in order to detect and to track emboli in cerebral arteries circulation[1]. The number of emboli detected is often linked to a vascular pathology, or to its degree of severity, like carotid stenosis.

Detection techniques available on current Doppler pulsed wave (PW) devices are based on backscattered energy between an embolus and background red blood cells. Time-frequency distributions, like spectrogram, are commonly used in order to compute embolic Doppler signal energy. Recently, a new alternative has been developed : it consists in modeling Doppler signal through a white noise filter using a two order autoregressive (AR) model [2]. Emboli occurrences appear as a model change. This technique is extended here to emboli sizing. Several approaches concerning emboli discrimination have been investigated: Doppler signal processing and parameters extraction [3], solid and gaseous emboli backscattered radiofrequency signal spectrum analysis [4], or two frequencies emission technique [5].

After a brief description of the sizing method, which consists in computing EBR (Embolus to Blood Ratio) values at two different emission frequencies, the parametric approach is presented. Comparison with Fourier technique results is then performed. Results have been processed and compared in terms of bias and standard deviations. Finally, multifrequency method is introduced to differentiate solid emboli from gaseous ones.

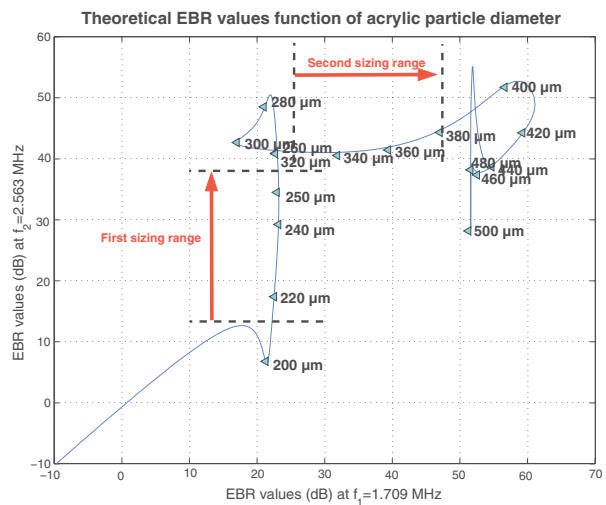

Fig. 1. Theoretical $E B R_{f_{2}}$ function of $E B R_{f_{1}}$

\section{EMBOLI SIZING TECHNIQUE}

The technique used here has been suggested by previous studies [6], [7] and [8]. An EBR value, embolus to blood ratio, is introduced in eq.(1). It explicitly represents the acoustic impedance mismatch between embolus and the surrounding red blood cells.

$$
\operatorname{EBR}\left(f, r_{e}\right)=\frac{\sigma_{e}}{\eta V}
$$

with $\sigma_{e}$ is the embolus backscatter embolus cross-section, a quite complex function essentially of emission frequency, embolus acoustical properties: size, composition (density $\rho$ and speed of sound $c$ ), computed supposing embolus spherically shaped. V is the volume of fluid flowing in Doppler sample volume, and $\eta$ is the blood backscatter coefficient.

This frequency dependance of embolus backscatter crosssection is applied in order to solid emboli size. A two frequencies emission PW system is so used for computing EBR values of emboli of different sizes. This fact is illustrated, in figure 1, which represents $E B R_{2}\left(f_{2}=2.563 \mathrm{MHz}\right)$ function of $E B R_{1}\left(f_{1}=1.709 \mathrm{MHz}\right)$. Emboli mimicking particles here are acrylic ones.

This curve shows the reliability of this technique, specially for two ranges of acrylic particles diameter (220 to $260 \mu \mathrm{m})$ and $(320$ to $380 \mu \mathrm{m})$. At a given size within these ranges, a single $\left(E B R_{1}, E B R_{2}\right)$ couple is allocated. Two energy estimators results are described below. 


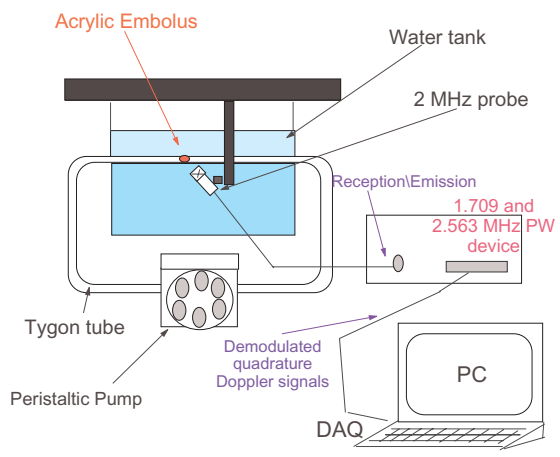

Fig. 2. Experimental set-up

\section{EXPERIMENTAL RESULTS}

\section{A. Experimental set-up}

In order to verify sizing technique, an in vitro set-up was made. It consists of a fluid, BMF (blood Mimicking Fluid) mainly consisting of $5 \mu \mathrm{m}$ orgasol particles, moving in a tygon tube with a peristaltic pump, which allows to generate pulsatile flow. Emboli were introduced by acrylic particles ranging from $220 \mu \mathrm{m}$ to $500 \mu \mathrm{m}$. The same experimental conditions were used for each measure: insonification angle around $\pi / 6$. Flow speed (near 7 to $10 \mathrm{~cm} . \mathrm{s}^{-1}$ ) is lower than those met in cerebral circulation, in order to avoid bubbles generated by the pump and the tube joins. The PW doppler device is a two frequencies 1.709 MHz and 2.563 MHz emission one. Emission power can be adjusted. Sample volume length and depth were fixed to 4 and $45 \mathrm{~mm}$ respectively, for all measures. The experimental set-up is represented in figure 2. The quadrature demodulated Doppler signals (for the two frequencies at two depths) were sampled at pulse repetition frequency (PRF) $6.4 \mathrm{kHz}$ using a data acquisition board (DAQ).

\section{B. Fourier Approach}

Energy estimation using Fourier approach is very common. It consists of power density calculations in frequency domain. As Doppler signal is strongly non-stationary, spectrogram technique is used. The EBR value is defined as, eq.(2):

$$
E B R=10 \log _{10}\left(0.5 * \frac{P_{E+B}}{P_{B}}\right)
$$

where $P_{E+B}$ and $P_{B}$ are respectively power backscattered by embolus in blood, and blood only. The figure 3 represents Fourier-based EBR values compared with theoretical ones, at each emission frequency.

Table I exhibits results (mean and standard deviation) obtained with Fourier approach at the two frequencies versus theoretical ones in brackets.

For acrylic sizes up to $400 \mu \mathrm{m}$, bias (over $10 \mathrm{~dB}$ ) can be mainly explained by signal saturations (magnitude out of dynamic range of PW device). Relatively significant bias

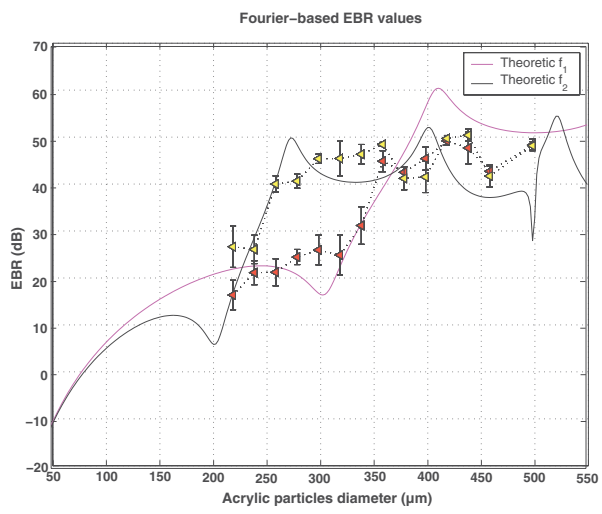

Fig. 3. $1.709 \mathrm{MHz}$ and $2.563 \mathrm{MHz}$ Fourier-based and theoretical EBR values function of acrylic particles diameter

TABLE I

FOURIER EVALUATED EBR MEAN AND STANDARD DEVIATION FOR ACRYLIC PARTICLES

\begin{tabular}{|c|c|c|}
\hline Size $(\mu \mathrm{m})$ & mean(theoretic) at $f_{1} ; f_{2}(\mathrm{~dB})$ & std $\left(f_{1}, f_{2}\right)(\mathrm{dB})$ \\
\hline 220 & $16.99(25.77) ; 27.28(20.65)$ & $3.13 ; 4.41$ \\
\hline 240 & $21.76(26.43) ; 26.71(32.51)$ & $2.56 ; 3.01$ \\
\hline 260 & $21.85(26.25) ; 40.66(44.61)$ & $2.87 ; 1.74$ \\
\hline 280 & $25.09(24.63) ; 41.27(52.22)$ & $1.61 ; 1.51$ \\
\hline 300 & $26.60(20.68) ; 46.05(42.42)$ & $3.15 ; 0.99$ \\
\hline 320 & $25.52(26.11) ; 46.11(44.57)$ & $4.25 ; 3.68$ \\
\hline 340 & $31.81(35.35) ; 47.01(44.30)$ & $4.01 ; 2.08$ \\
\hline 360 & $45.54(42.92) ; 49.11(45.22)$ & $2.23 ; 0.44$ \\
\hline
\end{tabular}

appeared close to small particles sizes, specially at emission frequency $f_{2}$. For example, for $220 \mu \mathrm{m}$ diameter acrylic particles, computed EBR values were higher than theoretical ones (around $10 \mathrm{~dB}$ for the two frequencies). Other energy computing approach was also introduced.

\section{Parametric Approach}

As previously enounced, Doppler signal can be modelled by a two order AR model. This signal can be expressed by eq.(3):

$$
y_{d}(n)=-\sum_{i=1}^{p} a_{i}(n) y_{d}(n-1)+u(n)
$$

with $\mathrm{p}$ is the model order, $a_{i}$ is $\mathrm{AR}$ coefficients and $\mathrm{u}(\mathrm{n})$ is a white noise. Power spectral density is then obtained from parameters $a_{i}$. The figure 4 represents EBR parametric estimated values compared with theoretical ones, at each emission frequency. Parametric approach results between 220 and $360 \mu \mathrm{m}$ were shown on table II. Unlike fourier computed values, EBR ratios, corresponding to 220 up to $320 \mu \mathrm{m}$ acrylic particles, presented low standard deviations (around 2-3 dB) and bias was not as important as previously at emission frequency $f_{2}$. 


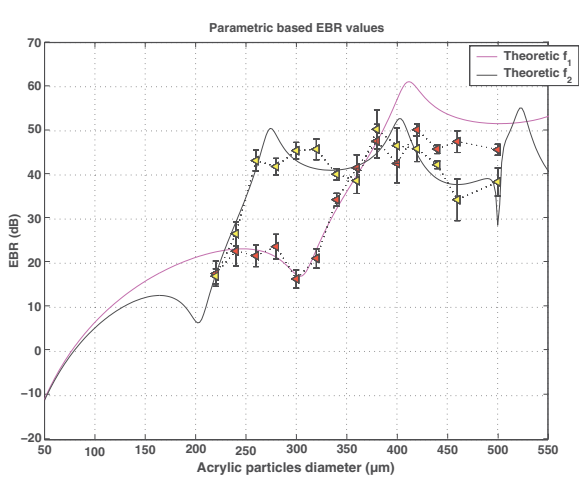

Fig. 4. $1.709 \mathrm{MHz}$ and $2.563 \mathrm{MHz}$ Parametric evaluated and theoretical EBR values function of acrylic particles diameter

TABLE II

PARAMETRIC EVALUATED EBR MEAN AND STANDARD DEVIATION FOR ACRYLIC PARTICLES

\begin{tabular}{|c|c|c|}
\hline Size $(\mu \mathrm{m})$ & mean(theoretic) at $\left(f_{1}, f_{2}\right)(\mathrm{dB})$ & std $\left(f_{1}, f_{2}\right)(\mathrm{dB})$ \\
\hline 220 & $17.58(25.77) ; 17.01(20.65)$ & $2.88 ; 1.69$ \\
\hline 240 & $22.67(26.43) ; 26.61(32.51)$ & $3.39 ; 2.75$ \\
\hline 260 & $21.63(26.25) ; 43.19(44.61)$ & $2.42 ; 2.38$ \\
\hline 280 & $23.71(24.63) ; 41.86(52.22)$ & $2.78 ; 1.85$ \\
\hline 300 & $16.37(20.68) ; 45.46(42.42)$ & $2.06 ; 1.87$ \\
\hline 320 & $21.04(26.11) ; 45.77(44.57)$ & $2.20 ; 2.33$ \\
\hline 340 & $34.33(35.35) ; 40.10(44.30)$ & $1.45 ; 1.30$ \\
\hline 360 & $41.53(42.92) ; 38.13(45.27)$ & $2.99 ; 2.88$ \\
\hline
\end{tabular}

\section{EMBOLI DIFFERENTIATION}

\section{A. Two frequencies technique}

As previously mentioned, the two frequencies PW device allowed us to estimate solid emboli sizes. This technique is now applied in order to differentiate gaseous emboli from solid ones. The parameter introduced here was $\Theta_{E B R}\left(f_{1}, f_{2}\right)$ which was expressed by eq.(4):

$$
\Theta_{E B R}\left(f_{1}, f_{2}\right)=E B R\left(f_{1}\right)-E B R\left(f_{2}\right)
$$

An important criteria of differentiation seems to be the evolution of the $\Theta_{E B R}\left(f_{1}, f_{2}\right)$ sign. Doppler signals attributed to $200 \mu \mathrm{m}$ air bubbles occurrences were recorded with the same two frequencies PW device. $\Theta_{E B R}\left(f_{1}, f_{2}\right)$ values were dressed in table III, they were always positive.

Backscattered Doppler signals of red blood cells aggregates, fat and gaseous emboli were then simulated. The first fre-

TABLE III

$\Theta_{E B R}\left(f_{1}, f_{2}\right)$ FOURIER AND PARAMETRIC VALUES FOR AIR BUBBLES

\begin{tabular}{|c|c|c|}
\hline Fourier & $\Theta_{E B R}\left(f_{1}, f_{2}\right)$ mean value $(\mathrm{dB})$ & std $(\mathrm{dB})$ \\
\hline & 12.40 & 2.71 \\
\hline Parametric & $\Theta_{E B R}\left(f_{1}, f_{2}\right)$ mean value $(\mathrm{dB})$ & std $(\mathrm{dB})$ \\
\hline & 15.80 & 2.92 \\
\hline
\end{tabular}

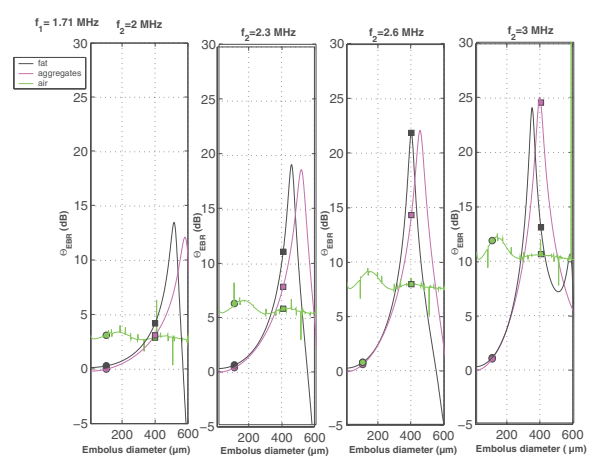

Fig. 5. $\Theta_{E B R}\left(f_{1}, f_{2}\right)$ function of emboli diameters and second frequency

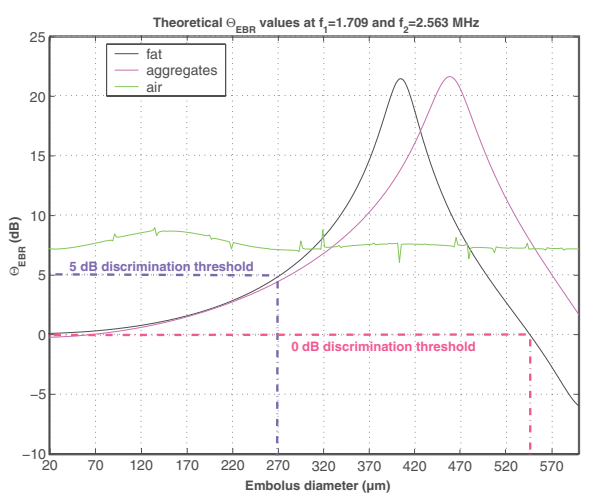

Fig. 6. $\Theta_{E B R}\left(f_{1}, f_{2}\right)$ function of emboli diameters at $f_{1}=1.709 \mathrm{MHz}$ and $f_{2}=2.563 \mathrm{MHz}$

quency was fixed at $f_{1}=1.709 \mathrm{MHz}$. Emboli sizes varied from 10 to $600 \mu \mathrm{m}$. Evolutions of $\Theta_{E B R}$ for these three emboli types where shown in figure 5 , with, for example, four frequency $f_{2} 2,2.3,2.6$ and $3 \mathrm{MHz}$. Simulations were performed with a $10 \mathrm{~mm}$ sample volume. Even if $\Theta_{E B R}\left(f_{1}, f_{2}\right)$ was always positive for air bubbles, this is not true in case of fat and aggregates: $\Theta_{E B R}$ was only negative for aggregates smaller than $40 \mu \mathrm{m}$, and for fat emboli up to $550 \mu \mathrm{m}$ at $f_{2}=$ 2.3 MHz. This parameter sign is not sufficient to discriminate solid and gaseous emboli. Nevertheless, if a threshold (around $5 \mathrm{~dB}$ ant not zero as previously) is established and the two emission frequencies were $f_{1}=1.709 \mathrm{MHz}$ and $f_{2}=2.563 \mathrm{MHz}$, $\Theta_{E B R}$ between gas and solid emboli difference could be made if diameters didn't exceed $270 \mu \mathrm{m}$, in figure 6 . Three frequencies approach was so suggested.

\section{B. Three frequencies technique}

Now, an approach was to simultaneously emit three frequencies. It was, also, to verify that $\Delta \Theta_{E B R}$ value sign, defined as eq.(5), is different with gaseous emboli $(>0)$ that for solid ones $(<0)$.

$$
\Delta \Theta_{E B R}=\Theta_{E B R}\left(f_{1}, f_{2}\right)-\Theta_{E B R}\left(f_{2}, f_{3}\right)
$$

Similar simulations, but now with three frequencies, were made with air bubbles, red blood cells aggregates, and fat 


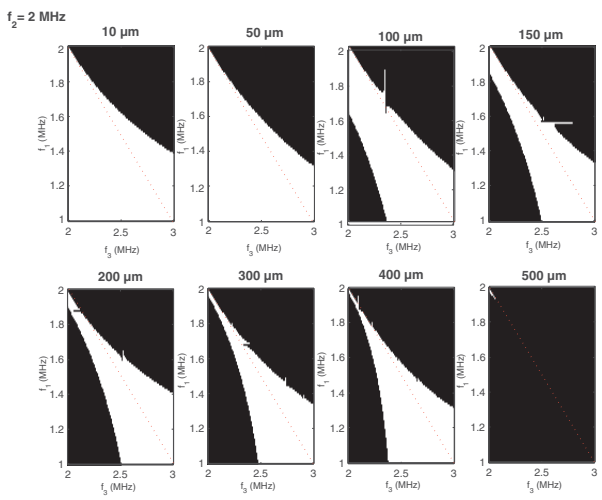

Fig. 7. $\Delta \Theta_{E B R}$ domains for air bubbles and fat emboli

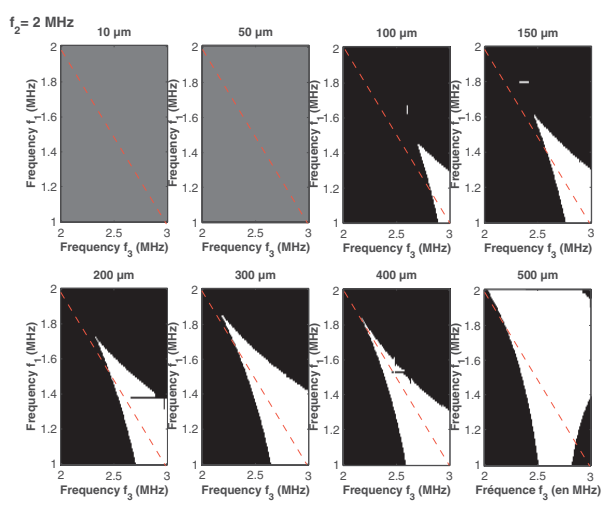

Fig. 8. $\Delta \Theta_{E B R}$ domains for air bubbles, fat and aggregates

emboli. Frequency $f_{2}$ is fixed at $2 \mathrm{MHz}$. Frequencies $f_{1}$ and $f_{3}$ were varied between 1 and $2 \mathrm{MHz}$, and 2 and $3 \mathrm{MHz}$ respectively. Emboli diameters range was contained between 10 and $600 \mu \mathrm{m}$. A binary representation was achieved in order to distinguish domains where $\Delta \Theta_{E B R}$ is positive for gaseous emboli and negative in case of solid ones. Figures 7 and 8 displayed these domains, respectively, by considering only fat, and red blood cells aggregates and fat for solid emboli. These figures showed $\Delta \Theta_{E B R}$ sign evolution, for example, for emboli diameters equals to $10,50,100,150,200$, 300, 400 and $500 \mu \mathrm{m}$. Regions, where $\Delta \Theta_{E B R}$ values were positive, colored white, or black in case of negative $\Delta \Theta_{E B R}$ values. When the total domain colored gray, $\Delta \Theta_{E B R}$ sign was identical for all computed $\left(f_{1}, f_{2}, f_{3}\right)$ triplet, and any differentiation were possible.

The frequencies triplet $\left(f_{1}, f_{2}, f_{3}\right)$, optimally chosen in order to have $\Delta \Theta_{E B R}$ negative and positive values for fat and air bubbles along a large size range, was, for example, 1.71, 2 and $2.32 \mathrm{MHz}$, in figure 7. Furthermore, these frequencies values must be relatively close to each other, so that a good transducer sensitivity was conserved. Nevertheless, if aggregates were taken account, in figure 8 ,conclusions were notably different. Thus, frequencies $\left(f_{1}, f_{3}\right)$ domain, which correspond to positive $\Delta \Theta_{E B R}$ values for air bubbles and negative for fat and aggregates, was smaller than previously. Frequency triplet previously introduced was true now only for aggregates up to $250 \mu \mathrm{m}$. Nevertheless, Clinical investigations were led to emboli sizes between 20 and $300 \mu \mathrm{m}$. An other frequencies couple $\left(f_{1}, f_{3}\right)$ must be introduced for smaller aggregates discrimination (up to $50 \mu \mathrm{m}$ ) 1.35 and $2.8 \mathrm{MHz}$. Compromise must be made between emboli size range discrimination and probe sensibility.

\section{CONCLUSION}

It was actually admitted that embolism consequences were correlated to embolus composition. That's why emboli differentiation and sizing remain promising researches. Approaches used in our study are multi-frequencies emission. The ability by a two frequencies PW device, associated with a good energy estimator, of sizing was proved. Nevertheless, this sizing was possible only on particular diameter ranges, if embolus composition was known. Parametric estimated EBR values presented less bias and standard deviation against theoretical than Fourier-based ones, specially in the two size ranges previously defined. Simulations showed practical attraction of multi-frequencies emission, concerning emboli differentiation. We have seen that the parameter $\Delta \Theta_{E B R}$ could be a discriminative factor in emboli discrimination, if emission frequencies were well chosen. Nevertheless, experimental measures must be performed.

\section{ACKNOWLEDGMENTS}

The authors would like to thank ATYS Médical for twofrequencies PW device making and French department of industry for this study financing.

\section{REFERENCES}

[1] M.P. Spencer, "Transcranial Doppler monitoring and causes of stroke from carotid endarterectomy.", Stroke, vol. 28 ,pp. 685-691, 1997.

[2] J-M. Girault, D. Kouamé, A. Ouahabi, and F. Patat, "Micro-emboli Detection : an ultrasound Doppler signal processing viewpoint.", IEEE Transactions on biomedical Engineering, vol. 47, pp 1431-1439,1999.

[3] J.L Smith, D. Evans, P. Bell and A.R. Naylor, "A comparison of four methods for distinguishing Doppler Signals from gaseous and particulate emboli.", Stroke, vol. 29, pp. 1133-1138, 1998.

[4] P. Palanchon, A. Bouakaz, J.H Van Blankestein, J. Klein, N. Born, and N. De Jong, "New technique for emboli detection and discrimination based on nonlinear charaacteristics of gas bubbles.", Ultrasound in Medicine and Biology, vol.27, pp. 801-808, 2001.

[5] R. Brucher and D. Russel, "Online automatic discrimination between solid and gaseous cerebral microemboli with the first multifrequency transcranial Doppler.", Stroke, vol. 33, pp. 1975-1980, 2002.

[6] M. Moehring, J.R Klepper, "Pulse Doppler Ultrasound Detection, Characterization and size estimation of emboli in flowing blood.", IEEE Transactions on biomedical Engineering, vol. 41, pp. 35-44, 1994.

[7] M. Moehring, J.A Ritcey, "Sizing emboli in blood flow using pulse Doppler ultrasound I: verification of the EBR model.", IEEE Transactions on biomedical Engineering, vol. 43, pp. 572-580, 1996.

[8] M. Moehring, J.A Ritcey and A. Ishimaru, "Sizing emboli in blood flow using pulse Doppler ultrasound II: Effects of beam refaction.", IEEE Transactions on biomedical Engineering, vol. 43, pp. 581-588, 1996. 